\title{
Hemangioendotelioma hepático infantil multifocal
}

\section{Multifocal hepatic hemangioendothelioma}

\author{
Carlos Zunino $^{\mathrm{a}}$, Maira Delgado ${ }^{\mathrm{b}}$, Gustavo Giachetto ${ }^{\mathrm{a}}$
}

${ }^{a}$ Clínica Pediátrica "C". Departamento de Pediatría, Facultad de Medicina. Universidad de la República. Uruguay

'Hospital de Tacuarembó, Administración de los Servicios de Salud del Estado, Uruguay

Recibido: 13 de septiembre de 2018; aceptado: 14 de febrero de 2019

\section{Resumen}

Introducción: El hemangioendotelioma hepático $(\mathrm{HEH})$ es un tumor benigno, raro en niños, que se presenta frecuentemente en el primer año de vida. La presentación clínica es variable y su diagnóstico se realiza en base a la sospecha clínica, estudios de laboratorio y de imagen. El objetivo fue describir un caso de hemangioendotelioma hepático multifocal. Caso clínico: Niña de 3 meses que presentó hepatomegalia sin elementos de falla hepática ni cardiaca. Se realizó ecografía y tomografía de abdomen que orientaron al diagnóstico de HEH que se confirmó con la angioresonancia de abdomen. Recibió glucocorticoides a altas dosis en forma prolongada. Al año y medio de iniciado el tratamiento se evidenció remisión del tumor. Presentó efectos secundarios por el tratamiento instaurado. Conclusiones: La presencia de una hepatomegalia aislada en un lactante asintomático debe hacer pensar en una probable patología tumoral, orientándonos por la clínica e imagenología al diagnóstico nosológico de la misma. Siempre se debe sospesar las posibles complicaciones con los riesgos de los tratamientos a realizar. En este caso la extensión del tumor y sus probables complicaciones justificó el uso de corticoterapia prologada a altas dosis a pesar de sus efectos adversos.

\begin{abstract}
Introduction: Hepatic hemangioendothelioma is a rare benign tumor in children, which frequently occurs in the first year of life. The clinical presentation is variable and the diagnosis is based on clinical suspicion, and laboratory and imaging studies. The objective was to describe a case of multifocal hepatic hemangioendothelioma. Clinical report: 3-month-old girl who presented hepatomegaly without elements of hepatic or heart failure. Abdominal ultrasound and CT scan were used to diagnose hepatic hemangioendothelioma, which was confirmed by CT abdominal angiography. The patient received glucocorticoid treatment at high doses for a prolonged period. A year and a half after treatment, there was evidence of tumor remission. She had side effects from the established treatment. Conclusions: In asymptomatic patients with isolated hepatomegaly, it should be considered a probable tumor pathology, considering the clinic and imaging studies. Possible complications and treatments risks must always be assessed. In this case, the tumor extension and its probable complications justified the use of prolonged corticosteroid therapy at high doses despite its adverse effects.
\end{abstract}

Palabras clave: Hepatomegalia; hemangioendotelioma; tumor hepático; niño

\section{Keywords:}

Hepatomegaly; hemangioendothelioma; child; liver tumor 


\section{Introducción}

El hemangioendotelioma hepático (HEH) es un tumor vascular, de origen mesenquimal, histológicamente benigno ${ }^{1,2}$. Se trata de una patología rara en niños, su incidencia es mayor en los dos primeros años de vida, presentándose la mayoría (más del $80 \%$ ) antes de los 6 meses de edad ${ }^{1,3,4}$. La presentación multifocal se observa en $45 \%$ de los casos ${ }^{1}$.

La presentación clínica es variable desde hepatomegalia asintomática a la insuficiencia cardíaca, siendo esta última de elevada mortalidad ${ }^{1,4,5}$. Puede presentarse con trombocitopenia por secuestro plaquetario y falla hepática. Se pueden asociar hemangiomas en otros sectores, sobre todo en piel ${ }^{1}$.

El diagnóstico se realiza en base a la sospecha clínica, estudios de laboratorio y de imagen ${ }^{2}$. La confirmación histológica no siempre se realiza dado los riesgos del procedimiento ${ }^{4}$.

En un alto porcentaje de casos regresan en forma espontánea alrededor de los 12-18 meses de realizado el diagnóstico, sin necesidad de tratamiento ${ }^{1,2}$. De requerir, existen diferentes tratamientos médicos o intervencionistas dependiendo del tumor, entre ellos se destacan: uso de corticoides, propanolol, quimioterapia, embolización arterial, hepatectomía parcial y trasplante hepático ${ }^{2-5,7-9}$.

El objetivo del presente artículo es describir la forma de presentación clínica e imagenológica, las opciones terapéuticas y la evolución de un caso de $\mathrm{HEH}$ multifocal en una lactante de 3 meses de edad.

\section{Caso clínico}

Tres meses, género femenino, procedente de medio deficitario rural de Uruguay, ingresa al Hospital Regional de Tacuarembó, por mal ascenso ponderal.

Producto de $7^{\circ}$ embarazo sin controles. Parto vaginal, 40 semanas de edad gestacional estimada, peso al nacer $3.220 \mathrm{~g}$, adecuada a la edad gestacional, talla 49 $\mathrm{cm}$, perímetro cefálico $34 \mathrm{~cm}$, vigorosa. Resultado de pesquisa neonatal normal, peso al egreso $3.000 \mathrm{~g}$. Alimentada a pecho directo exclusivo hasta antes del mes de vida, luego continua con leche de vaca entera sin agregado de azúcar. No recibe vitamina D. Desarrollo psicomotor normal. Inmunizaciones vigentes. Screening materno negativo para: síflis, virus de la hepatitis B y virus de la inmunodeficiencia humana (VIH).

$\mathrm{Al}$ ingreso peso $4.480 \mathrm{~g}$ (menor a percentil 3), talla $58 \mathrm{~cm}$ (percentil 15). Al examen: vigil, reactiva, apirética, eupneica. Piel y mucosas sin palidez ni lesiones. Panículo adiposo disminuido. A nivel abdominal límite superior hepático en $5^{\text {to }}$ espacio intercostal derecho, borde inferior a $4 \mathrm{~cm}$ del reborde costal de consistencia normal, indoloro. No presenta esplenomegalia. No se palpan otras tumoraciones abdominales, ni en territorios ganglionares. Examen pleuropulmonar y cardiovascular normal.

Se solicitaron pruebas hepáticas (niveles de aminotransaminasas séricas, bilirrubina, fosfatasa alcalina, glutamiltranspeptidasa) y pruebas de coagulación (tiempo de protrombina, tiempo de tromboplastina parcial) que fueron normales. Se realizó ecografía de abdomen que mostró hígado aumentado de tamaño, ecoestructura heterogénea con pequeñas imágenes hipoecoicas difusas en lóbulo derecho e izquierdo.

Tomografía computada (TC) de abdomen y pelvis con y sin contraste (figura 1): marcada hepatomegalia de densidad heterogénea a expensas de múltiples lesiones nodulares hipodensas que ocupan ambos lóbulos de tamaño entre 2 y $18 \mathrm{~mm}$. Luego de administrar contraste intravenoso realzan en fase arterial y persisten de mayor densidad que el parénquima hepático en fase portal. No se evidenciaron adenomegalias, tumoraciones abdominales ni pelvianas. Resto del estudio normal.

Se planteó probable HEH multifocal, solicitándose resonancia nuclear magnética (RNM) con angiografía de abdomen que evidenció hepatomegalia difusa, el parénquima hepático está sustituido por pequeñas lesiones focales redondeadas de entre 5 y $11 \mathrm{~mm}$ en gran número, son bien delimitadas, hipointensas en T1, hiperintensas en $\mathrm{T} 2$, y con realce intenso con gadolinio, con relleno centrípeto (figura 2). Las imágenes son compatibles con HEH infantil multifocal.

Se decide iniciar tratamiento con corticoides (prednisolona) a dosis de $3 \mathrm{mg} / \mathrm{kg} /$ día vía oral por 6 meses, con posterior descenso gradual de dosis, continuando al año con hidrocortisona vía oral a dosis fisiológicas. Se realizó seguimiento ambulatorio clínico (oncólogo, cardiólogo y pediatra) y ecográfico cada 3 meses. Se controló con nueva RNM con angiografía de abdomen al año y medio de iniciado el tratamiento con remisión de las lesiones hepáticas (figura 3). Presentó complicaciones por corticoterapia en altas dosis por tiempo prolongado: insuficiencia suprarrenal, síndrome de Cushing y talla baja. No presentó otras complicaciones.

\section{Discusión}

La desnutrición en el primer trimestre de vida de esta niña, que motivó el ingreso hospitalario, fue catalogada de causa exógena ya que recibía alimentación hipocalórica y la curva ponderal mejoró con la corrección del aporte dietético. Esto permitió alejar la posibilidad de una patología que asociara la falla de crecimiento con la hepatomegalia encontrada en el examen físico como hallazgo. En este caso, la hepato- 


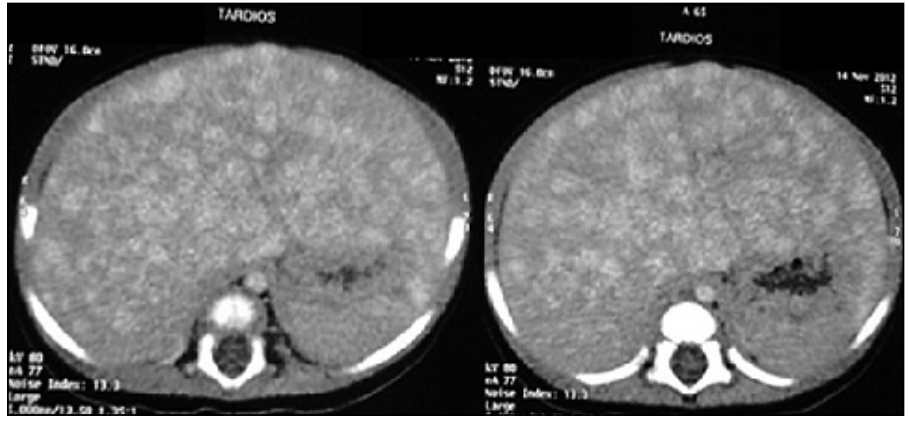

Figura 1. Tomografía de abdomen: múltiples nódulos hipodensos en todo el parénquima hepático.

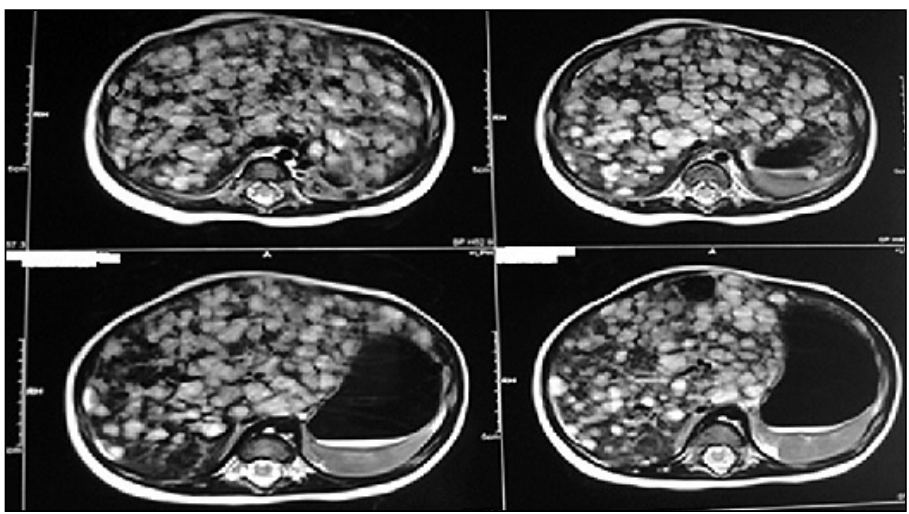

Figura 2. Resonancia con angiografía de abdomen. Hepatomegalia y lesiones focales que realzan con gadolinio.

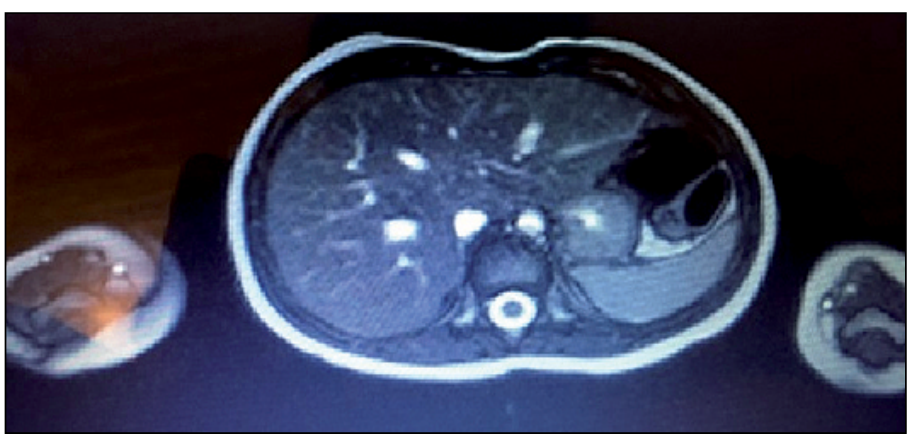

Figura 3. Resonancia con Angiografía de abdomen a los 18 meses del tratamiento. Franca reducción del número y tamaño de las lesiones.

megalia era de consistencia normal e indolora y no se acompañaba de alteraciones en el examen físico ni con otros síntomas asociados. Frente a una lactante con hepatomegalia aislada las causas pueden ser diversas: inflamación (infecciones, tóxicos), depósito (glucogenosis, Enfermedad de Gaucher, Niemann-Pick, déficit de alfa 1 antitripsina), infiltración (tumores hepáticos benignos y malignos, metástasis de tumores extrahepáticos), congestión vascular (enfermedad venooclu- siva, síndrome de Budd-Chiari, insuficiencia cardíaca congestiva) y obstrucción biliar (atresia biliar, quiste de colédoco $)^{10}$. Por frecuencia y gravedad, las primeras etiologías a descartar son las infecciosas, enfermedades metabólicas de depósito y fibrosis quística ${ }^{11}$.

Esta niña presentaba hepatomegalia asintomática y aislada, sin elementos de lesión, ni de disfunción hepática. Contando con serología para virus de hepatitis B y VIH negativa y la pesquisa neonatal normal, en ausencia de elementos clínicos que permitieran sospechar otras patologías, fue prioritario descartar los tumores hepáticos. Los tumores hepáticos más frecuentes son las metástasis de neuroblastoma o nefroblastoma ${ }^{1}$. Los tumores primarios son menos frecuentes, los de mayor incidencia son tumores malignos $(60 \%)$ como hepatoblastoma y hepatocarcinoma. Los tumores benignos como el hemangioendotelioma son raros y su diagnóstico se realiza en base a las características clínicas, estudios analíticos y de imagen ${ }^{2,11}$.

En esta paciente los hallazgos clínicos que sugirieron el diagnóstico de HEH son la edad(menor de 6 meses), el sexo femenino y la presentación como hepatomegalia aislada ${ }^{1-4}$. El 50\% de los casos de estos tumores se presentan de esta forma ${ }^{3}$. Es importante destacar que otras de las manifestaciones iniciales de este tumor son la falla cardíaca congestiva, con un $70 \%$ de mortalidad, la trombocitopenia por secuestro plaquetario y más raro falla hepática ${ }^{1}$. Siendo estas también posibles complicaciones que surgen en la evolución, por lo que deben ser evaluadas con un correcto seguimiento, para su pesquisa y eventual tratamiento oportuno ${ }^{2}$. Esta paciente no presentó estas manifestaciones ni al inicio ni en la evolución.

Los estudios imagenológicos son importantes en la evaluación de este tipo de casos. La ecografía abdominal es una herramienta útil, siendo el primer estudio imagenológico a realizar frente a toda sospecha de tumor hepático, dado el bajo costo, inocuidad y buen rendimiento. Sin embargo, en muchas oportunidades es necesario otros estudios radiológicos: tomografía computada y angioresonancia que aporten otros hallazgos más específicos que ayuden en la orientación diagnóstica ${ }^{12}$. La paciente del caso presentó las imágenes características que permitieron el diagnóstico de HEH. Si bien con cualquiera de las técnicas imagenológicas es posible realizar el diagnóstico, la de mayor rendimiento es la angioresonancia. La mayoría de los casos no es necesario la confirmación histológica ${ }^{2,4}$. En esta paciente se decidió no realizar biopsia hepática dado el alto riesgo de sangrado ${ }^{4}$ y la claridad de los hallazgos imagenológicos.

No existen protocolos estandarizados internacionales, ni nacionales para el tratamiento de este tipo de tumor $^{8}$. En la literatura se han comunicado series con muy pocos pacientes ${ }^{3,9}$. Las opciones terapéuticas de- 
penden de las características del tumor, si es solitario o multifocal, la presencia o ausencia de síntomas y la presencia o no de complicaciones ${ }^{1-3,13}$. A pesar que un alto porcentaje de los casos de $\mathrm{HEH}$ revierten en forma espontánea entre los 12 y 18 meses de realizado el diagnóstico ${ }^{1}$, en esta paciente se decidió iniciar tratamiento por el compromiso difuso del parénquima hepático. En estos casos existe riesgo de que las lesiones rápidamente se transformen en sintomáticas con riesgo de complicaciones que pueden causar la muerte ${ }^{2,4}$. La mayoría de los autores recomiendan la corticoterapia en la fase proliferativa, a dosis de 2 a $3 \mathrm{mg} / \mathrm{kg} /$ día durante un periodo aproximado de 5 meses $^{4,5,14}$. En este caso, se realizó 6 meses de prednisolona a dosis plenas y luego se fue descendiendo gradualmente la dosis hasta completar el año de tratamiento. El 30\% tiene una respuesta total, $40 \%$ una respuesta parcial, requiriendo un tratamiento adicional y un $30 \%$ no responden ${ }^{4}$. En esta paciente la respuesta fue muy favorable, recibiendo únicamente corticoides. Se plantearon otras alternativas terapéuticas como el uso de propanolol, INF alfa y la vincristina ${ }^{3,4,7,8,14}$. Al analizar el balance riesgo - beneficio de cada uno de estos tratamientos, se consideró que el mejor para esta paciente era el uso de corticoides.

Como opciones intervencionistas se destacan la embolización arterial hepática, hepatectomía parcial y el trasplante hepático ${ }^{3,4}$. No se consideraron de primera elección en esta paciente dada su edad, las características clínicas del tumor (multifocalidad) y la baja experiencia en nuestro medio en cuanto al manejo de este tipo de tumores. En este caso no se planteó el tratamiento quirúrgico, se lo debe considerar en algunos tumores solitarios ${ }^{1,3}$.

Se hace evidente la necesidad de seguir y controlar las eventuales complicaciones propias del tumor y las derivadas del tratamiento. En la bibliografía revisada si bien se sugieren algoritmos diagnósticos y terapéuticos ${ }^{3,7,15}$ no está protocolizada la forma de seguimiento y control de estos pacientes. En este caso se llevó a cabo trimestralmente evaluando la clínica, analítica y estudios imagenológicos. Fue importante el control de los efectos adversos de la corticoterapia a altas dosis por tiempo prolongado. La intervención de un equipo multidisciplinario fue fundamental para lograr mejores resultados ${ }^{3,14}$.

\section{Conclusiones}

La clínica e imagenología permitieron el diagnóstico de un tumor hepático raro, benigno pero que puede tener complicaciones muy severas. Siempre debe sospesar las posibles complicaciones con los riesgos de los tratamientos a realizar. En este caso la remisión completa del tumor justificaría las complicaciones causadas por la corticoterapia prologada a altas dosis.

\section{Responsabilidades Éticas}

Protección de personas y animales: Los autores declaran que los procedimientos seguidos se conformaron a las normas éticas del comité de experimentación humana responsable y de acuerdo con la Asociación Médica Mundial y la Declaración de Helsinki.

Confidencialidad de los datos: Los autores declaran que han seguido los protocolos de su centro de trabajo sobre la publicación de datos de pacientes.

Derecho a la privacidad y consentimiento informado: Los autores han obtenido el consentimiento informado de los pacientes y/o sujetos referidos en el artículo. Este documento obra en poder del autor de correspondencia.

\section{Conflicto de intereses}

Los autores declaran no tener conflicto de intereses. 


\section{Referencias}

1. Ferreira H, Archila M.

Hemangioendotelioma hepático infantil: características clínicas e imagenológicas. Presentación de un caso y revisión de la literatura. The Free Library 2006;9(2):1515.

2. Araújo AR, Maciel I, Costa JC, Vieira A, Enes C, Santos Silva E. Hemangioendotelioma hepático infantil. Un caso multifocal y bilobular, asintomático, con regresión espontánea. An Pediatr (Barc) 2008;68(5):507-10.

3. Eun Hee Kim, Kyung Nam Koh, Meerim Park, Bo Eun Kim, Ho Joon Im, Jong Jin Seo. Clinical features of infantile hepatic hemangioendothelioma. Korean J Pediatr 2011;54(6):260-6.

4. Leal N, López Santamaría M, Gámez M, Murcia J, López Gutiérrez J, Larrauri J, et al. Hemangioendotelioma multifocal hepático infantil. ¿Es siempre un tumor benigno?. Cir Pediatr 2004;17(1):8-11.

5. Al-Tonbary Y, Fouda A. Infantile hepatic hemangioendothelioma: an 8-month old infant successfully treated with a corticosteroid. Hematol Oncol Stem Cell Ther. 2009;2(3):422-5.

6. Wang T, Wang Y, Liang

Y, Lu G. Infantile Hepatic

Hemangioendothelioma associated with congestive heart failure. Two case reports with different outcomes. Medicine 2015;94(52):1-4.

7. Gnarra M, Behr G, Kitojewski A, Wu J, Anupindi S, Shawber C, et al. History of the infantile hepatic hemangioma: from imaging to generating a differential diagnosis. World J Clin Pediatr 2016;5(3):273-80.

8. Palacios J, Carnasquel V, Jaramillo E, Herrera D. Hemangioma hepático infantil tratado con propanolol. Rev Mex Cir Ped 2014;18(3):130-9.

9. Maaloul I, Aloulou H, Hentati Y, Kamoun T, Mnif Z, Hachicha M. Infantile hepatic hemangioendothelioma successfully treated by low dose of propranolol. La Presse Médicale 2017;46(4):454-6.

10. Muñoz G. Hepatomegalia. Pediatr
Integral 2011;15 (3):221-37.

11. Fernández I, De Diego E, Trugeda M, Sandoval F. Masas abdominales en la infancia. Bol Pediatr 2001;41(176):122130.

12. Chiorean L, Xin-Wu Cui, Tannapfel A, Franke D, Stenzel M, Kosiak W, et al. Benign liver tumors in pediatric patients Review with emphasis on imaging features. World J Gastroenterology 2015;21(28):8541-61.

13. Betül Sevinir, Tanju Özkan. Infantile hepatic hemangioendothelioma: Clinical presentation and treatment. Turk J Gastroenterol 2007;18(3):182-7.

14. López R, López JC, Belendez C, Herrero A, Mateos M, Ramírez G. Tumores vasculares en la infancia. An Pediatr(Barc) 2010;72(2):1-15.

15. Kuroda T, Kumagai M, Nosaka S, Nakazawa A, Takimoto T, Hoshino K. Critical infantile hepatic hemangioma: results of a nationwide survey by the Japanese Infantile Hepatic Hemangioma Study Group. Journal of Pediatric Surgery 2011;46:2239-43. 